\title{
Atherogenic index of plasma is related to coronary atherosclerotic disease in elderly individuals: a cross-sectional study
}

\author{
Haomin Huang ${ }^{1+}$, Xiaolong Yu ${ }^{2 \dagger}$, Lamei Li ${ }^{1}$, Ganwei Shi ${ }^{1}$ Feng $\mathrm{Li}^{1}$, Jianqiang Xiao ${ }^{1}$, Zhihua Yun ${ }^{3}$ and Gaojun Cai ${ }^{1 *}$ (D)
}

\begin{abstract}
Background: Dyslipidaemia plays an important role in coronary atherosclerotic disease (CAD). The relationship between the atherogenic index of plasma (AIP) and CAD in elderly individuals was explored in this study.

Methods: Elderly individuals (age $\geq 65$ years) who underwent coronary angiography from January 2016 to October 2020 were consecutively enrolled in the study.

Results: A total of 1313 individuals, including 354 controls (non-CAD) and 959 CAD patients, were enrolled. In univariate analysis of all populations, the adjusted AIP (aAIP) in the CAD group was $1.13(0.96,1.3)$, which was significantly higher than that in the controls $[1.07(0.89,1.26)]$. However, in subgroup analyses, this phenomenon was only present in males. In addition, further study showed that aAIP was positively related to CAD severity. In binary logistic regression analyses, after adjusting for sex, age, smoking status, primary hypertension (PH), type 2 diabetes mellitus (T2DM), heart rate (HR), white blood cell (WBC) and platelet (PLT), AIP remained independently related to CAD in elderly individuals and was superior to traditional and other nontraditional lipid indices. Subgroup analyses showed that AIP independently influenced CAD risk in males. Ultimately, sensitivity analyses were performed excluding all coronary emergencies, and the final results were similar.

Conclusions: AIP was positively related to the risk and severity of CAD in elderly individuals and was superior to traditional and other nontraditional lipid profiles. However, this association only exists in elderly males.
\end{abstract}

Keywords: Atherogenic index of plasma, Coronary atherosclerotic disease, Elderly, Lipid metabolism, Atherosclerosis, Cross-sectional study

\section{Background}

With the accelerating pace of modern life and the development of social economics, people limit physical activity and eat more fat than before, resulting in coronary atherosclerotic disease (CAD), which is the primary cause of death worldwide $[1,2]$. There are approximately 11 million CAD patients in China [3]. Among all

\footnotetext{
*Correspondence: cgj982@126.com

${ }^{\dagger}$ Haomin Huang and Xiaolong Yu contributed equally to this work. 'Department of Cardiology, Wujin Hospital Affiliated with Jiangsu University, The Wujin Clinical College of Xuzhou Medical University, Changzhou City, Jiangsu Province, China

Full list of author information is available at the end of the article
}

casualties, elderly individuals bear the majority of the CAD burden $[4,5]$. In addition, as life expectancy has increased, elderly individuals will gradually become a large part of the whole population, and approximately one-fifth of the world's population will be older than 65 years of age by 2030 [6].

Elevated low-density lipoprotein cholesterol (LDL-C) increases the risk of CAD, but the risk of cardiovascular events remains after standardized lipid-lowering therapy [7]. Therefore, exploring a comprehensive blood lipid index that can better predict and evaluate CAD has become a research hotspot. 
Various comprehensive lipid indices have been previously identified as associated with CAD. Among them, atherogenic index of plasma (AIP), which is expressed as logarithmic transformation of the quotient of triglycerides (TG) divided by high-density lipoprotein cholesterol (HDL-C) $[\lg (\mathrm{TG} / \mathrm{HDL}-\mathrm{C})]$, is a new reliable and predictable CAD index that has been verified in various populations, including Chinese Han people [8], very early onset CAD patients ( $\leq 35$ years of age) [9] and type 2 diabetes mellitus (T2DM) patients after stent implantation [10]. The ability of AIP to predict CAD was stronger than other atherosclerosis indices, such as non-high-density lipoprotein cholesterol (non-HDL-C), which is calculated as TC minus HDL-C (TC - HDL-C); LDL-C/HDL-C; non-HDL-C/HDL-C, which is known as the atherogenic index (AI); and total cholesterol (TC) * TG * LDL-C/ HDL-C, which is known as the lipoprotein combine index (LCI).

However, whether AIP could successfully predict the presence of CAD remains controversial [11-13]. Moreover, a previous study showed that elderly patients had obviously different clinical characteristics than young patients with acute myocardial infarction [14]. Whether AIP is related to CAD risk in elderly individuals has not been verified. In view of this information, this hospitalbased cross-sectional study was conducted to explore the correlation between AIP and CAD in elderly individuals.

\section{Methods}

\section{Participants}

Elderly individuals ( $\geq 65$ years of age) who underwent coronary angiography (CAG) in a third-tier general hospital from January 2016 to October 2020 were consecutively selected. The exclusion criteria were as follows: 1 ) individuals with serious liver dysfunction [alanine aminotransferase (ALT) $\geq 200 \mathrm{U} / \mathrm{L}$ ] or renal insufficiency [estimated glomerular filtration rate (eGFR) $<45 \mathrm{ml} /$ $\mathrm{min} / 1.73 \mathrm{~m}^{2}$ ], hyperthyroidism or hypothyroidism; 2) individuals using lipid metabolism drugs; 3) individuals with a history of cancer; and 4) individuals with repeated CAG and incomplete lipid profile data. Finally, a total of 1313 elderly participants, including 354 non-CAD controls and 959 CAD patients, were enrolled in this study. Figure 1 presents the flowchart outlining the study.

This study adhered to the tenants of the Declaration of Helsinki and was approved by the Institutional Ethics Committee of Wujin Hospital (Ethics approval number: 201606). Because this cross-sectional study was retrospectively designed, written informed consent was not needed.

\section{Diagnostic criteria}

All subjects were examined by CAG via radial or femoral using the Judkin technique, and the CAG images were assessed by two experienced cardiologists who were blinded to the study. World Health Organization diagnostic criteria were used to diagnose CAD with at least one main coronary artery exhibiting $\geq 50 \%$ stenosis [15], and the severity of CAD was evaluated according to the number of main coronary arteries $\geq 50 \%$ stenosis. The control group was defined as individuals with main coronary artery stenosis $<50 \%$. Primary hypertension $(\mathrm{PH})$ and T2DM were defined according to the characteristics described in a previous study [16]. In short, $\mathrm{PH}$ was defined as repeated systolic blood pressure (SBP) $\geq 140$ $\mathrm{mmHg}$ and/or diastolic blood pressure (DBP) $\geq 90$ $\mathrm{mmHg}$ at least three times on different days or previously diagnosed PH. T2DM was defined as two measures of fasting blood glucose $\geq 7.0 \mathrm{mmol} / \mathrm{L}$ and/or random glucose level $\geq 11.1 \mathrm{mmol} / \mathrm{L}$ plus symptoms or previously diagnosed T2DM. A smoker was defined as

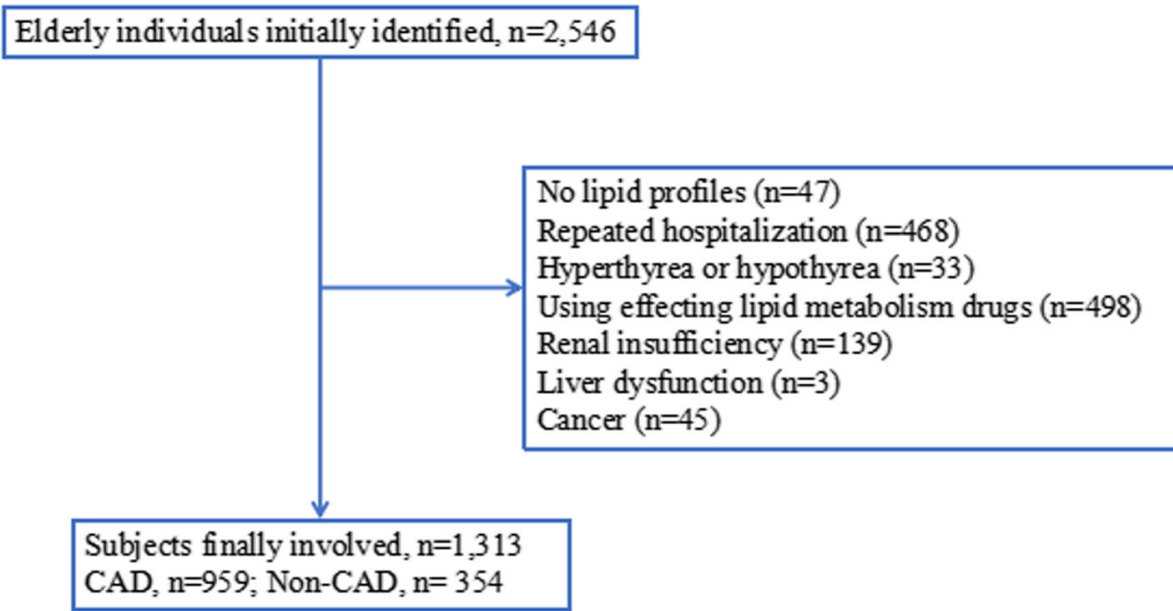

Fig. 1 Flow chart describing the procedure of subjects involved in this study 
an individual who smokes more than ten cigarettes per day, and a drinker was defined as an individual who intakes more than $40 \mathrm{ml}$ of alcohol per day.

\section{Data collection}

After fasting for $12 \mathrm{~h}$, venous blood was obtained from each participant. General data, including sex, age, height, weight, SBP, DBP, heart rate (HR), PH, T2DM, smoking status, and drinking status, were extracted from medical records. Biochemical parameters, including white blood cell (WBC), platelet (PLT), serum uric acid (SUA), eGFR, and lipid profiles, were analysed using an automated biochemical analyser.

Body mass index (BMI) was expressed as the quotient of weight divided by height squared (weight/height ${ }^{2}$ ). To avoid negative AIP values, TG/HDL-C was multiplied by 10 , and then the logarithmic value was obtained as the adjusted AIP (aAIP) [lg(TG/HDL-C"10)].

\section{Statistical analyses}

SPSS 22.0 software (SPSS Inc., IL, USA) and Stata 12.0 software were used to analyse all data. Missing values were replaced by the median value. Continuous variables distributed normally were expressed as the means \pm standard deviation and compared using Student's t-tests, and those distributed abnormally were expressed as median and interquartile range (IQR) and compared using Mann-Whitney $U$ test. The Kolmogorov-Smirnov test was used to evaluate the normality of the data. Categorical variables were assessed by Chi-square test. The correlations between aAIP and age, BMI, SUA and LDL-C were performed using Spearman correlation analysis. All variables were explored at four aAIP levels base on the median and IQR. The relationship between aAIP and the risk of CAD was explored using binary logistic regression analyses expressed as odds ratio (OR) with $95 \%$ confidence intervals $(95 \% \mathrm{CI})$. In the model of all individuals, OR was adjusted for sex, age, smoking status, PH, T2DM, HR, WBC and PLT. In the elderly male

Table 1 Clinical characteristics of whole participants

\begin{tabular}{|c|c|c|c|}
\hline Characteristics & Control group $(n=354)$ & CAD group $(n=959)$ & $P$ \\
\hline Male, n(\%) & $183(51.7)$ & $610(63.6)$ & $<0.001$ \\
\hline Age, year & $70(67,74)$ & $71(68,75)$ & 0.001 \\
\hline $\mathrm{BMI}, \mathrm{kg} / \mathrm{m}^{2}$ & $24.57(22.67,27.10)$ & $24.39(22.41,26.42)$ & 0.067 \\
\hline Smoker, n(\%) & $86(24.3)$ & $316(33)$ & 0.003 \\
\hline Drinker, n(\%) & $45(12.7)$ & $108(11.3)$ & 0.467 \\
\hline $\mathrm{PH}, \mathrm{n}(\%)$ & $248(70.1)$ & $788(78.1)$ & 0.003 \\
\hline T2DM, n(\%) & $79(22.3)$ & $313(32.6)$ & $<0.001$ \\
\hline SBP, mmHg & $139(128,150)$ & $140(127,152)$ & 0.215 \\
\hline $\mathrm{DBP}, \mathrm{mmHg}$ & $80(71,86)$ & $80(72,89)$ & 0.192 \\
\hline $\mathrm{HR}, \mathrm{BPM}$ & $70(66,80)$ & $72(67,80)$ & 0.031 \\
\hline \multicolumn{4}{|c|}{ Laboratory parameters } \\
\hline $\mathrm{WBC}, 10^{9} / \mathrm{L}$ & $5.97(5,7.06)$ & $6.79(5.52,8.43)$ & $<0.001$ \\
\hline $\mathrm{PLT}, 10^{9} / \mathrm{L}$ & $187.5(155,228.25)$ & $196(162,236)$ & 0.016 \\
\hline eGFR, $\mathrm{ml} / \mathrm{min}$ & $75.09(63.54,89.23)$ & $74.24(60.95,87.13)$ & 0.179 \\
\hline SUA, umol/L & $347.3(291,426.2)$ & $347(286.6,415)$ & 0.369 \\
\hline $\mathrm{TC}, \mathrm{mmol} /$ & $4.28(3.68,4.91)$ & $4.42(3.72,5.11)$ & 0.044 \\
\hline $\mathrm{TG}, \mathrm{mmol} / \mathrm{L}$ & $1.38(1,1.99)$ & $1.43(1.01,1.98)$ & 0.379 \\
\hline $\mathrm{HDL}-\mathrm{C}, \mathrm{mmol} / \mathrm{L}$ & $1.15(0.98,1.36)$ & $1.07(0.93,1.24)$ & $<0.001$ \\
\hline LDL-C, mmol/L & $2.64(2.15,3.22)$ & $2.88(2.27,3.45)$ & 0.001 \\
\hline aAIP & $1.07(0.89,1.26)$ & $1.13(0.96,1.3)$ & 0.007 \\
\hline Non-HDL-C & $3.09(2.48,3.69)$ & $3.32(2.64,3.99)$ & $<0.001$ \\
\hline LDL-C/HDL-C & $2.28(1.75,2.96)$ & $2.66(2.09,3.34)$ & $<0.001$ \\
\hline $\mathrm{Al}$ & $2.6(2.04,3.4)$ & $3.05(2.38,3.91)$ & $<0.001$ \\
\hline LCl & $13.42(7.69,25.23)$ & $16.77(9.3,30.58)$ & $<0.001$ \\
\hline
\end{tabular}

CAD coronary atherosclerotic disease, BMI body mass index, PH primary hypertension, T2DM type 2 diabetes mellitus, SBP systolic blood pressure, DBP diastolic blood pressure, HR heart rate, BPM beats per minute, WBC white blood cell, PLT platelet, eGFR estimated glomerular filtration rate, SUA serum uricacid, TC total cholesterol, TG triglyceride, HDL-C high-density lipoprotein cholesterol, LDL-C low-density lipoprotein cholesterol, aAIP adjusted atherogenic index of plasma, nonHDL-C non-high-density lipoprotein cholesterol, Al atherogenic index, LCl lipoprotein combine index 
model, OR was adjusted for alcohol consumption, T2DM, WBC, PLT, and SUA. In the elderly female model, OR was adjusted for age, PH, T2DM, HR and WBC. A two-sided $P$-value $<0.05$ was considered statistically significant.

\section{Results}

\section{Clinical characteristics of all participants}

As shown in Table 1, it was obvious that age and the prevalence of males were higher in the CAD group compared with the controls [age, $71(68,75)$ vs $70(67,74)$, $P<0.05$; males, $63.6 \%$ vs $51.7 \%, P<0.001$ ]. Elderly individuals with $C A D$ had a significantly higher prevalence of smoking, $\mathrm{PH}$ and T2DM compared with controls (all $P<0.05)$. In addition, CAD individuals had higher levels of HR, WBC, PLT, TC, LDL-C, aAIP, non-HDL-C, LDLC/HDL-C, AI and LCI and had lower levels of HDL-C than those of the controls (all $P<0.05$ ). As for aAIP, it's levels continually increased with the aggravation of $C A D$ showed in Fig. 2. Nevertheless, the proportion of drinkers and BMI, SBP, DBP, eGFR, SUA and TG levels were not significantly different between the two groups (all $P>0.05$ ).

\section{Factors influencing aAIP}

As shown in Fig. 3, in both the CAD and control groups, aAIP was higher in females, and subjects with $\mathrm{PH}$ or T2DM also had higher levels of aAIP than those without (all $P<0.05$ ). However, it was surprising that the aAIP levels were not significantly different in smoking and non-smoking individuals. As shown in Fig. 4, Spearman correlation analysis indicated that aAIP was significantly positively associated with BMI, SUA and LDL-C. However, aAIP was negatively associated with age (all $P<$ 0.05).

As shown in Table 2, all variables were compared in four aAIP levels based on median and IQR. The results

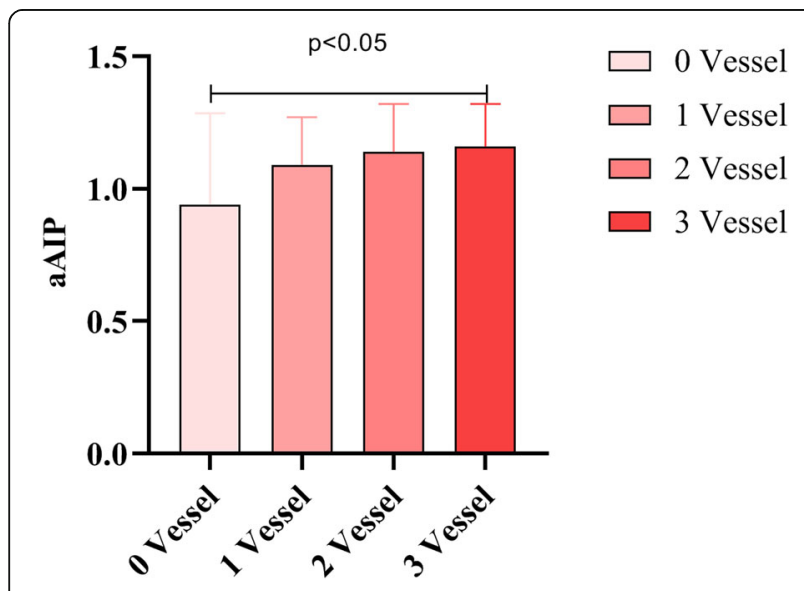

Fig. 2 The relationship between aAIP and the severity of CAD revealed that the proportion of $\mathrm{CAD}$, males, drinkers, $\mathrm{PH}$, and T2DM and the levels of BMI, SBP, DBP, PLT, eGFR, SUA, TC, LDL-C, HDL-C, TG, non-HDL-C, LDL-C/HDL-C, AI and LCI were significantly different at the four levels of aAIP (all $P<0.05$ ).

\section{Subgroup analyses}

Subgroup analyses showed that clinical characteristics differed between the two sexes. Among elderly males, individuals with CAD had higher PLT and aAIP levels but had a lower prevalence of alcohol consumption and a lower level of SUA than those of the controls. In elderly females, no differences were noted between the two groups. In contrast, the prevalence of $\mathrm{PH}$ and the levels of age, SBP, HR and TC were higher in CAD individuals than in the controls (Table 3 in supply materials).

\section{Multivariate logistic regression analysis}

Multivariate logistic regression analyses were executed in all subjects, elderly males and elderly females to identify the risk factors for CAD. As represented in Fig. 5, aAIP remained a risk factor for CAD in elderly individuals after adjusting for sex, age, smoking status, $\mathrm{pH}$, T2DM, HR, WBC and PLT (OR 1.75; 95\% CI: 1.06-2.88, $P<0.05)$ and was superior to traditional or other nontraditional lipid indices. In subgroup analyses, aAIP remained a dependent risk factor for CAD in elderly males after adjusting for confounding factors (OR 2.42; 95\% CI: $1.2-4.88, P<0.05)$. Conversely, in the elderly female model, aAIP was not an independent risk factor for CAD after adjusting for confounding factors $(P>0.05)$ (Fig. 5). Further more, a sensitivity analysis excluding coronary emergency showed a similar results (Table 4 in supply materials).

\section{Discussion}

The present study discovered that AIP was independently and positively related to the presence and severity of CAD in elderly individuals and was superior to traditional and other nontraditional lipid indices. However, subgroup analyses did not supply any evidence to support this conclusion in elderly females.

Coronary atherosclerosis is the pathological basis of $\mathrm{CAD}$, and lipid deposition is the leading cause of coronary atherosclerosis. Among them, LDL-C is a chief causal factor [17], and its cumulative exposure is a larger risk factor for $\mathrm{CAD}$, especially in elderly individuals, even independent of a single LDL-C level [18]. Accordingly, decreasing LDL-C levels can reduce cardiovascular events $[19,20]$.

As reported in a previous study, small dense lowdensity lipoprotein (sdLDL) is a component of LDL particles with a small volume and high density [21]. Compared to other LDL, sdLDL is more easily oxidized and 


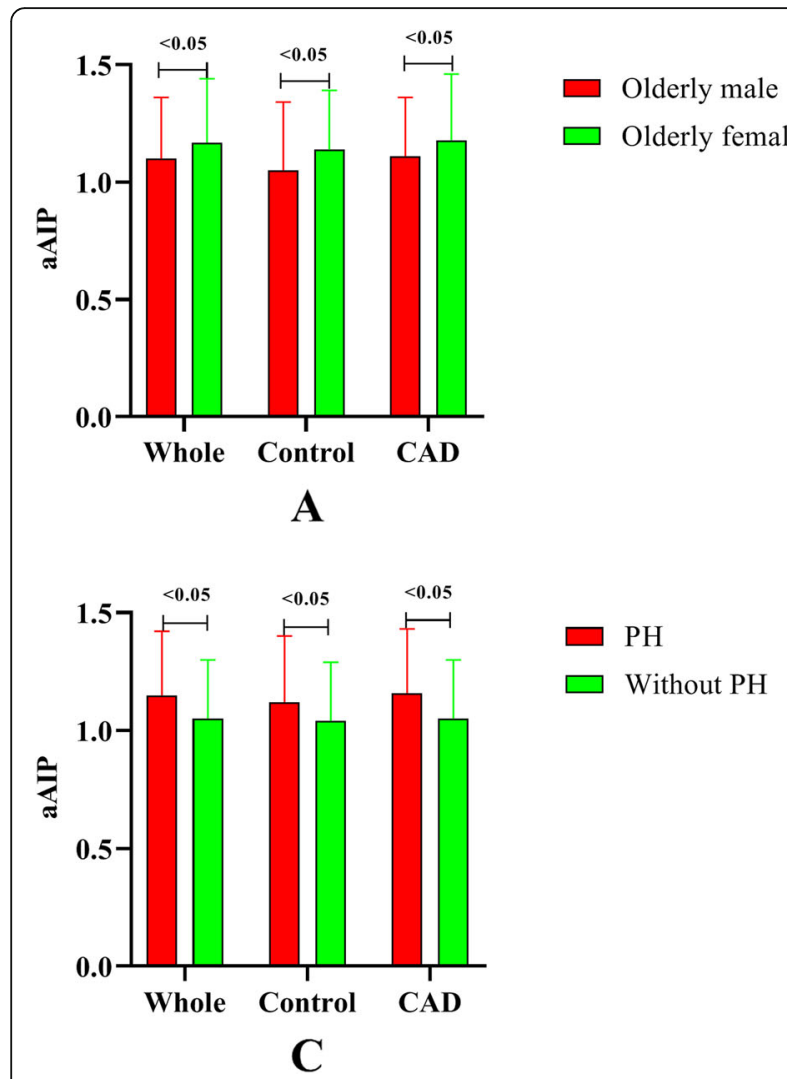

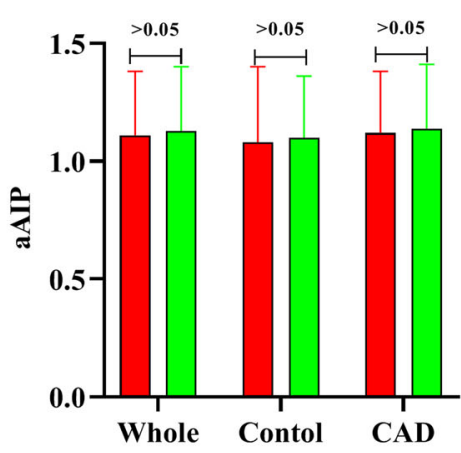

B

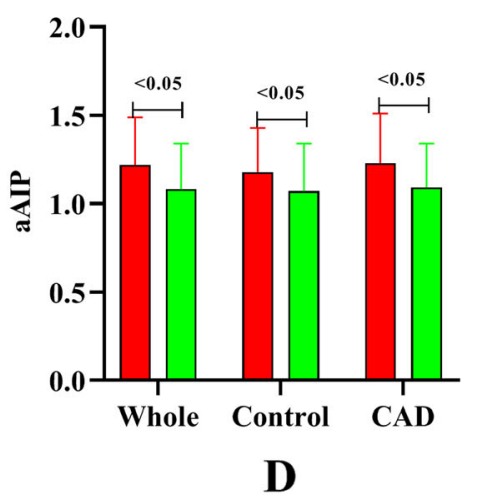

Smoking

No smoking

Fig. 3 Influence factors of aAIP. A, Gender; B, Smoking; C, PH; D, T2DM

damages vascular endothelial cells. Then, sdLDL passes through cells, resulting in cholesterol deposition and ultimately lead to atherosclerosis [22]. As a result, lipidinduced atherosclerosis could be comprehensively assessed by combining routine blood lipids and sdLDL [23]. Nevertheless, the procedure of testing sdLDLs is so complex and expensive that it cannot be widely used in clinical practice. Therefore, we need to identify a simple method to indirectly measure LDL particle size.

Dobiásová et al. [24] introduced the conception of AIP first in 2001, reporting that it had better clinical application value than the single traditional lipid index. Subsequently, an increasing amount of evidence showed that AIP was negatively correlated with LDL particle size and can generally indicate sdLDL size [25]. Afterwards, AIP was gradually defined to be related to T2DM [26], abdominal obesity [27], non-alcoholic fatty liver [28], arterial stiffness [29] and other factors. Recently, a hospitalbased study involving 3600 individuals who underwent CAG reported that AIP could reliably diagnose CAD with a sensitivity of $76.4 \%$ and specificity of $61.8 \%$ [30]. Furthermore, another study found that AIP could be regarded as a cardiovascular risk factor in Mexicans aged 18 to 22 years [31], which was similar to findings from a previous study in China [9].
However, the correlation between AIP and CAD in elderly individuals (age $\geq 65$ years) has not been well established. Moreover, whether AIP can reliably predict the risk of CAD in elderly females has been controversial. On the one hand, a study including 4644 postmenopausal women suggested that AIP might independently predict CAD in postmenopausal women [11]. On the other hand, Nansseu JR et al. [12] found that AIP might not independently impact the risk of CAD among Cameroonian postmenopausal women. Similarly, another study reported that AIP did not significantly increase the risk of acute coronary syndrome in females (average age $64.9 \pm 10$ years) [32]. Therefore, this study analysed the association between AIP and the risk of CAD in elderly individuals, a special group who were at high risk of having CAD. In addition, because the role of AIP in predicting $\mathrm{CAD}$ in elderly females is controversial, subgroup analysis by sex was conducted to evaluate the correlation between AIP and CAD.

Why does the difference in the relationship between AIP and CAD still exist in the two sexes, even though females in this age stage are minimally influenced by oestrogen? Several factors must be taken into account to answer this question. First, Fig. 3 shows that median AIP levels in elderly females were significantly higher than 

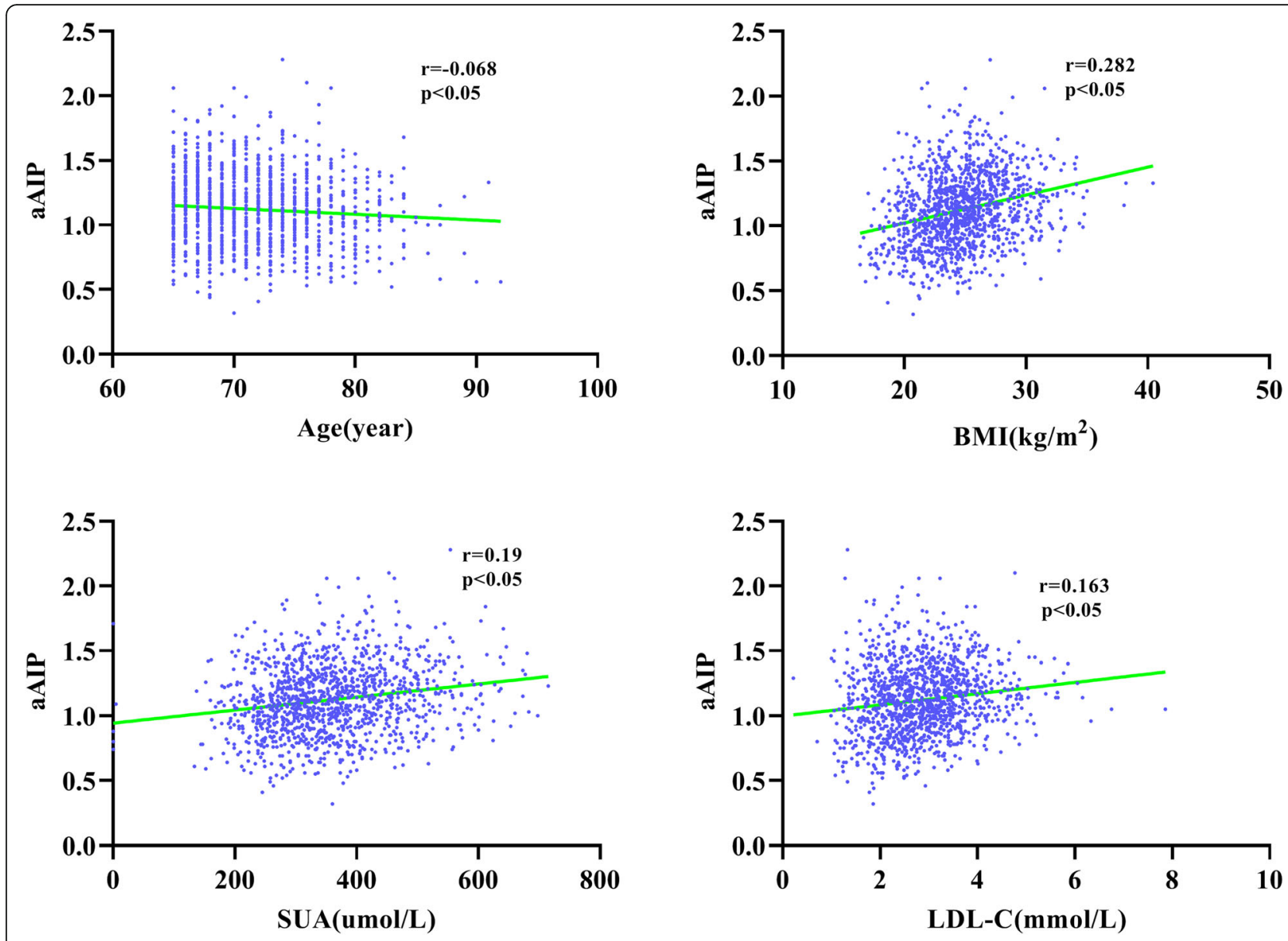

Fig. 4 Linear correlation analysis of aAIP with age, BMI, SUA, and LDL-C

that in males, which might play an important role in accounting for this difference. In addition, PLT levels, which might influence the level of AIP according to Table 2, differed between the CAD group and controls in elderly males rather than elderly females. Indeed, some physical data, such as exercise and diet, that influence TG or HDL-C could not be acquired in this study, which may confound the results.

In addition, this study found that the levels of SUA, BMI, WBC, PLT, eGFR, LDL-C and other lipid indices and the proportion of patients with $\mathrm{PH}$ and $\mathrm{T} 2 \mathrm{DM}$ was closely related to AIP, and all of these factors were considered risk factors for CAD in a previous study [6, 33$36]$. In view of this relationship, both elderly males and females should be encouraged to adhere to a healthy lifestyle to reduce AIP levels, even if the association between AIP and CAD risk was not established in elderly females.

\section{Strength}

This present study has some following strengths. First, the subjects of this study were elderly individuals (age $\geq$
65 years), a group for whom controversy exists regarding the relationship between AIP and CAD risk. This study calculated aAIP levels in elderly individuals with different numbers of main coronary artery stenoses. Then, the correlation between aAIP and other risk factors for CAD was also analysed. Moreover, subgroup analysis stratified by sex was conducted.

\section{Limitation}

Some limitations have to be taken into account. First, due to the cross-sectional and retrospective design, the cause-result effect cannot be well interpreted. In the future, prospective and cohort studies should be conducted to verify the results. Second, as a retrospective study, some data related to AIP, such as waist circumference, diet and physical labour, could not be acquired in the analysis, which might interfere with the final results. Furthermore, the individuals included in controls were not truly healthy, and their representativeness needs to be further verified. Finally, the Gensini score could not be used to assess the severity of coronary stenosis. However, the number of diseased coronary arteries was used 
Table 2 Clinical Characteristics in four different aAIP levels

\begin{tabular}{|c|c|c|c|c|c|}
\hline Characteristics & aAIP $<0.94(n=321)$ & $0.94 \leq \mathrm{aAIP}<1.12(n=335)$ & $1.12 \leq$ aAIP $<1.285(n=329)$ & aAIP $\geq 1.285(n=328)$ & $P$ \\
\hline$\overline{C A D}, \mathrm{n}(\%)$ & $214(66.7)$ & $240(71.6)$ & $254(77.2)$ & $251(76.5)$ & 0.008 \\
\hline Male, n(\%) & $221(68.8)$ & $202(60.3)$ & $192(58.4)$ & $178(54.3)$ & 0.002 \\
\hline Age, year & $71(68,75)$ & $71(68,75)$ & $71(67,75)$ & $70(67,73.75)$ & 0.085 \\
\hline $\mathrm{BMI}, \mathrm{kg} / \mathrm{m}^{2}$ & $23.36(21.09,25.32)$ & $24.22(22.49,26.04)$ & $24.74(22.86,26.67)$ & $25.47(23.44,27.66)$ & $<0.001$ \\
\hline Smoker, n(\%) & $105(32.7)$ & $105(31.3)$ & $93(28.3)$ & $99(30.2)$ & 0.653 \\
\hline Drinker, n(\%) & $50(15 . .6)$ & $31(9.3)$ & $44(13.4)$ & $28(8.5)$ & 0.013 \\
\hline $\mathrm{PH}, \mathrm{n}(\%)$ & $221,567)$ & $239(71.3)$ & $263(79.9)$ & $279(85.1)$ & $<0.001$ \\
\hline T2DM, n(\%) & $49(15.3)$ & $89(26.6)$ & $116(35.3)$ & $138(42.1)$ & $<0.001$ \\
\hline $\mathrm{SBP}, \mathrm{mmHg}$ & $136(124.5,150)$ & $140(123,150)$ & $140(130,152.5)$ & $140(130,152)$ & 0.01 \\
\hline $\mathrm{DBP}, \mathrm{mmHg}$ & $80(70,86.5)$ & $80(72,86)$ & $80(73,89.5)$ & $81(74,89.75)$ & 0.012 \\
\hline $\mathrm{HR}, \mathrm{BPM}$ & $72(65,80)$ & $72(66,80)$ & $72(68,81)$ & $72(68,80)$ & 0.26 \\
\hline \multicolumn{6}{|c|}{ Laboratory parameters } \\
\hline WBC, $10^{9} / \mathrm{L}$ & $6.24(5.05,7.94)$ & $6.46(5.36,8.07)$ & $6.49(5.41,8.03)$ & $6.68(5.65,8.02)$ & 0.071 \\
\hline $\mathrm{PLT}, 10^{9} / \mathrm{L}$ & $183.5(150,222.5)$ & $198(160,232)$ & $193(161,236.5)$ & $202(169,238.75)$ & $<0.001$ \\
\hline eGFR, ml/min & $71.19(59.95,85.55)$ & $73.48(60.87,86.81)$ & $74.62(61.01,87.66)$ & $77.62(64.87,90.83)$ & 0.008 \\
\hline SUA, umol/L & $325.05(268.25,390)$ & $334.3(273,400)$ & $365(300,430)$ & $368(309.6,437.15)$ & $<0.001$ \\
\hline $\mathrm{TC}, \mathrm{mmol} / \mathrm{L}$ & $4.16(3.56,4.78)$ & $4.36(3.71,5.01)$ & $4.45(3.7,5.23)$ & $4.62(3.89,5.31)$ & $<0.001$ \\
\hline $\mathrm{TG}, \mathrm{mmol} / \mathrm{L}$ & $0.85(0.7,0.97)$ & $1.22(1.05,1.38)$ & $1.65(1.45,1.89)$ & $2.57(2.14,3.31)$ & $<0.001$ \\
\hline $\mathrm{HDL}-\mathrm{C}, \mathrm{mmol} / \mathrm{L}$ & $1.33(1.16,1.52)$ & $1.14(1.01,1.28)$ & $1.04(0.93,1.15)$ & $0.94(0.82,1.04)$ & $<0.001$ \\
\hline LDL-C, mmol/L & $2.51(2.04,3.07)$ & $2.93(2.33,3.39)$ & $3.03(2.32,3.61)$ & $2.9(2.3,3.52)$ & $<0.001$ \\
\hline Non-HDL-C & $2.76(2.30,3.37)$ & $3.23(2.62,3.76)$ & $3.39(2.70,4.10)$ & $3.66(2.97,4.31)$ & $<0.001$ \\
\hline LDL-C/HDL-C & $1.89(1.48,2.33)$ & $2.48(2.03,3.04)$ & $2.85(2.25,3.48)$ & $3.12(2.53,3.66)$ & $<0.001$ \\
\hline Al & $2.12(1.68,2.56)$ & $2.74(2.32,3.36)$ & $3.27(2.59,3.95)$ & $3.88(3.28,4.52)$ & $<0.001$ \\
\hline $\mathrm{LCl}$ & $6.53(4.13,9.25)$ & $13.4(9.25,18.23)$ & $20.49(13.75,30.32)$ & $38.4(24.64,56.9)$ & $<0.001$ \\
\hline
\end{tabular}

CAD coronary atherosclerotic disease, BMI body mass index, PH primary hypertension, T2DM type 2 diabetes mellitus, SBP systolic blood pressure, DBP diastolic blood pressure, HR heart rate, BPM beats per minute, WBC white blood cell, PLT platelet, eGFR estimated glomerular filtration rate, SUA serum uricacid, aAIP adjusted atherogenic index of plasma, TC total cholesterol, TG triglyceride, HDL-C high-density lipoprotein cholesterol, LDL-C low-density lipoprotein cholesterol, non-HDL-C non-high-density lipoprotein cholesterol, Al atherogenic index, LCl lipoprotein combine index

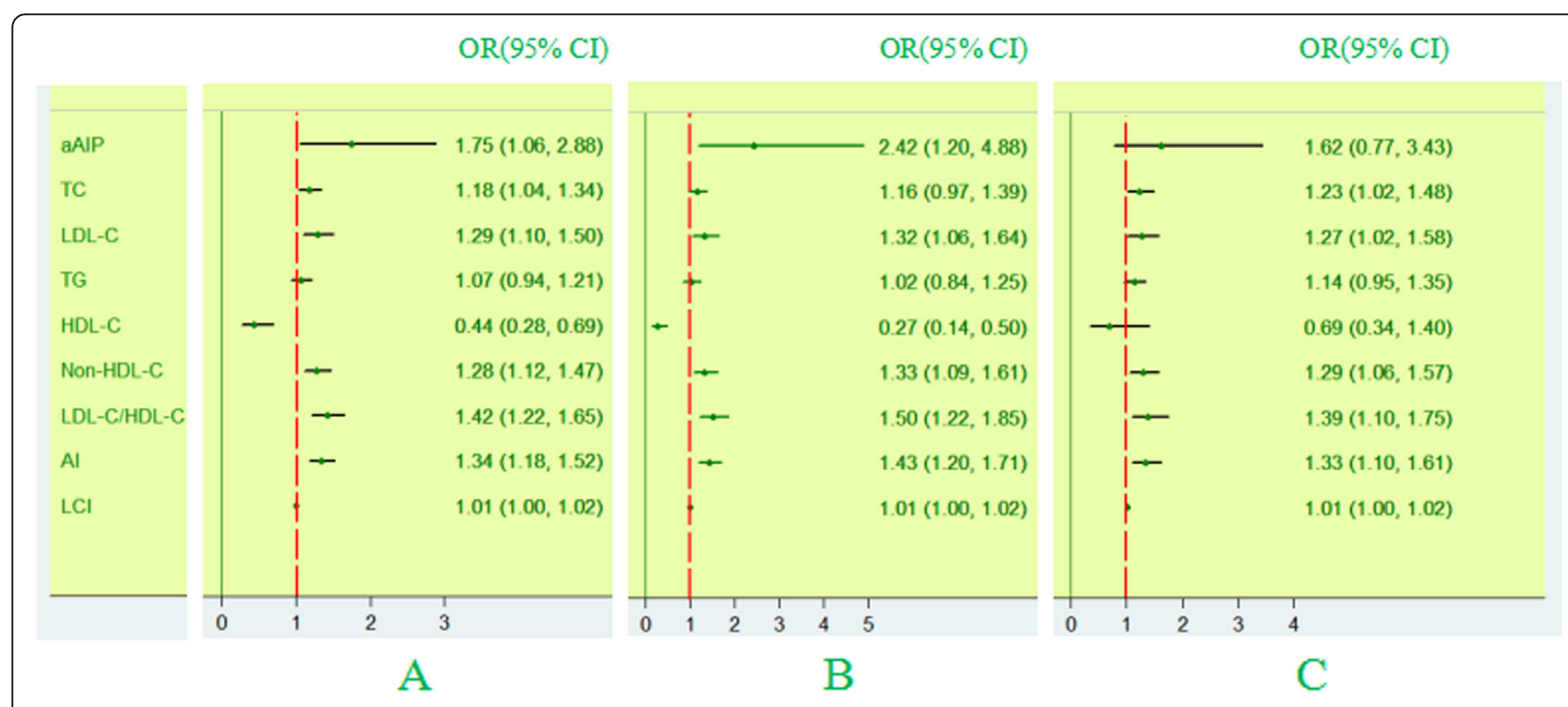

Fig. 5 Forest plot of multivariate logistic regression analyses of CAD risk factors. A, in whole population; B, in elderly males; $C$, in elderly females 
to evaluate the correlation between AIP and the severity of $\mathrm{CAD}$ according to the medical records.

\section{Conclusion}

AIP was independently and positively related to the risk and severity of CAD in elderly males but not in elderly females, which might be superior to traditional and other nontraditional lipid profiles. In the future, AIP may be used in the clinic to predict and prevent CAD as early as possible in elderly males, especially those with risk factors for CAD.

\begin{abstract}
Abbreviations
CAD: coronary atherosclerotic disease; CAG: coronary angiography; BMl: body mass index; PH: primary hypertension; T2DM: Type 2 diabetes mellitus; HR: heart rate; BPM: beats per minute; SBP: systolic blood pressure; DBP: diastolic blood pressure; WBC: white blood cell; ALT: alanine aminotransferase; eGFR: estimated glomerular filtration rate; PLT: platelet; SUA: serum uricacid; aAIP: adjusted atherogenic index of plasma; TC: total cholesterol; HDL-C: high-density lipoprotein cholesterol; non-HDL-C: nonhigh-density lipoprotein cholesterol; LDL-C: low-density lipoprotein cholesterol; TG: triglyceride; Al: atherogenic index; LCI: lipoprotein combine index; OR: odds ratio; 95\% Cl: 95\% confidence interval; IQR: interquartile range
\end{abstract}

\section{Supplementary Information}

The online version contains supplementary material available at https://doi. org/10.1186/s12944-021-01496-8.

Additional file 1:. Table S3 Clinical characteristics in elderly males and females. Table 54 Multivariate logistic regression analysis of individuals after excluding coronary emergency.

\section{Acknowledgements}

We thank all our colleagues at the Department of Cardiology.

\section{Authors' contributions}

Gaojun Cai conceived and designed the experiments; Haomin Huang and Xiaolong Yu performed the experiments; Lamei Li, Ganwei Shi and Feng Li analyzed the data; Zhihua Yun and Jianqiang Xiao contributed regents/ materials/analysis tools; Haomin Huang and Xiaolong Yu wrote the paper. The authors read and approved the final manuscript.

\section{Funding}

This study was supported by grants from Changzhou science and technology support program(Grant No. CE20195003) and Xuzhou Medical University (Grant No. XYFM2020009) to Gaojun Cai.

\section{Availability of data and materials}

The datasets of this study are available from the corresponding author.

\section{Declarations}

\section{Consent of publication}

Not applicable.

\section{Ethics approval and consent to participate}

The research protocol was approved by the Clinical Research Ethics Committee of Wujin Hospital, Jiangsu University (Ethics approval number: 201606). Because of the cross-sectional study design, informed consent was not needed.

\section{Competing interests}

The authors declare that they have no competing interests..

\section{Author details}

'Department of Cardiology, Wujin Hospital Affiliated with Jiangsu University, The Wujin Clinical College of Xuzhou Medical University, Changzhou City, Jiangsu Province, China. ${ }^{2}$ Department of Ultrasound, Wujin Hospital Affiliated with Jiangsu University, The Wujin Clinical College of Xuzhou Medical University, Changzhou City, Jiangsu Province, China. ${ }^{3}$ Department of Clinical Laboratory, Wujin Hospital Affiliated with Jiangsu University, The Wujin Clinical College of Xuzhou Medical University, Changzhou City, Jiangsu Province, China.

Received: 12 April 2021 Accepted: 29 June 2021

Published online: 11 July 2021

\section{References}

1. Arnett DK, Blumenthal RS, Albert MA, Buroker AB, Goldberger ZD, Hahn EJ, et al. 2019 ACC/AHA guideline on the primary prevention of cardiovascular disease: a report of the American College of Cardiology/American Heart Association task force on clinical practice guidelines. Circulation. 2019; 140(11):e596-646. https://doi.org/10.1161/CIR.0000000000000678.

2. Blumenthal D, Hsiao W, Blumenthal DM. Caring for coronary artery disease in China: managing modernization. JAMA Intern Med. 2016;176(4):521-3. https://doi.org/10.1001/jamainternmed.2016.0198.

3. Ma LY, Chen WW, Gao RL, Liu LS, Zhu ML, Wang YJ, et al. China cardiovascular diseases report 2018: an updated summary. J Geriatr Cardiol. 2020;17(1):1-8. https://doi.org/10.11909/j.issn.1671-5411.2020.01.001.

4. Orkaby AR, Driver JA, Ho YL, Lu B, Costa L, Honerlaw J, et al. Association of Statin use with all-Cause and Cardiovascular Mortality in US veterans 75 years and older. JAMA. 2020;324(1):68-78. https://doi.org/10.1001/jama.2020. 7848 .

5. Kovacic JC, Moreno P, Hachinski V, Nabel EG, Fuster V. Cellular senescence, vascular disease, and aging: part 1 of a 2-part review. Circulation. 2011; 123(15):1650-60. https://doi.org/10.1161/CIRCULATIONAHA.110.007021.

6. Heidenreich PA, Trogdon JG, Khavjou OA, Butler J, Dracup K, Ezekowitz MD, et al. Forecasting the future of cardiovascular disease in the United States: a policy statement from the American Heart Association. Circulation. 2011; 123(8):933-44. https://doi.org/10.1161/CIR.0b013e31820a55f5.

7. Matsuura Y, Kanter JE, Bornfeldt KE. Highlighting residual atherosclerotic cardiovascular disease risk. Arterioscler Thromb Vasc Biol. 2019;39(1):e1-9.

8. Cai G, Shi G, Xue S, Lu W. The atherogenic index of plasma is a strong and independent predictor for coronary artery disease in the Chinese Han population. Medicine (Baltimore). 2017;96(37):e8058. https://doi.org/10.1097/ MD.0000000000008058.

9. Cai G, Liu W, Lv S, Wang X, Guo Y, Yan Z, et al. Gender-specific associations between atherogenic index of plasma and the presence and severity of acute coronary syndrome in very young adults: a hospital-based observational study. Lipids Health Dis. 2019;18(1):99. https://doi.org/10.1186/ s12944-019-1043-2.

10. Qin Z, Zhou K, Li Y, Cheng W, Wang Z, Wang J, et al. The atherogenic index of plasma plays an important role in predicting the prognosis of type 2 diabetic subjects undergoing percutaneous coronary intervention: results from an observational cohort study in China. Cardiovasc Diabetol. 2020; 19(1):23. https://doi.org/10.1186/s12933-020-0989-8.

11. Guo Q, Zhou S, Feng X, Yang J, Qiao J, Zhao Y, et al. The sensibility of the new blood lipid indicator--atherogenic index of plasma (AIP) in menopausal women with coronary artery disease. Lipids Health Dis. 2020;19(1):27. https://doi.org/10.1186/s12944-020-01208-8.

12. Nansseu JR, Moor VJ, Nouaga ME, Zing-Awona B, Tchanana G, Ketcha A. Atherogenic index of plasma and risk of cardiovascular disease among Cameroonian postmenopausal women. Lipids Health Dis. 2016;15(1):49. https://doi.org/10.1186/s12944-016-0222-7.

13. Wu TT, Gao Y, Zheng YY, Ma YT, Xie X. Atherogenic index of plasma (AIP): a novel predictive indicator for the coronary artery disease in postmenopausal women. Lipids Health Dis. 2018;17(1):197. https://doi.org/1 0.1186/s12944-018-0828-Z.

14. Huang J, Qian HY, Li ZZ, Zhang JM. Comparison of clinical features and outcomes of patients with acute myocardial infarction younger than 35 years with those older than 65 years. Am J Med Sci. 2013;346(1):52-5. https://doi.org/10.1097/MAJ.0b013e318265e33e.

15. Nomenclature and criteria for diagnosis of ischemic heart disease. Report of the Joint International Society and Federation of Cardiology/World Health 
Organization task force on standardization of clinical nomenclature. Circulation. 1979:59(3):607-9.

16. Cai G, Zhang B, Ma C, Shi G, Weng W, Xue S. Associations of Rs3744841 and Rs3744843 polymorphisms in endothelial lipase Gene with risk of coronary artery disease and lipid levels in a Chinese population. PLoS One. 2016;11(9): e0162727. https://doi.org/10.1371/journal.pone.0162727.

17. Gragnano F, Calabrò P. Role of dual lipid-lowering therapy in coronary atherosclerosis regression: evidence from recent studies. Atherosclerosis. 2018;269:219-28. https://doi.org/10.1016/j.atherosclerosis.2018.01.012.

18. Song YJ, Du X, Zheng MY, Liu YC, Ma YH, Li HX, et al. A prospective cohort study of the effect of cumulative exposure to low density lipoprotein cholesterol on new-onset acute myocardial infarction. Chinese Circulation Journal. 2020;35(3):246-53.

19. Cesaro A, Bianconi V, Gragnano F, Moscarella E, Fimiani F, Monda E, et al. Beyond cholesterol metabolism: the pleiotropic effects of proprotein convertase subtilisin/kexin type 9 (PCSK9). Genetics, mutations, expression, and perspective for long-term inhibition. Biofactors. 2020;46(3):367-80 https://doi.org/10.1002/biof.1619.

20. Calabrò $\mathrm{P}$, Gragnano F, Pirro M. Cognitive function in a randomized trial of Evolocumab. N Engl J Med. 2017;377(20):1996-7. https://doi.org/10.1056/ NEJMc1712102.

21. Teramoto T, Sasaki J, Ishibashi S, Birou S, Daida H, Dohi S, et al. Cardiovascular disease risk factors other than dyslipidemia. Executive summary of the Japan atherosclerosis society (JAS) guidelines for the diagnosis and prevention of atherosclerotic cardiovascular diseases in Japan-2012 version. J Atheroscler Thromb. 2013;20(10):733-42. https://doi. org/10.5551/jat.17368.

22. Kwon SW, Yoon SJ, Kang TS, Kwon HM, Kim JH, Rhee J, et al. Significance of small dense low-density lipoprotein as a risk factor for coronary artery disease and acute coronary syndrome. Yonsei Med J. 2006;47(3):405-14. https://doi.org/10.3349/ymj.2006.47.3.405.

23. Yildiz G, Hür E, Özçiçek A, Candan F, Kayatas M. The mean platelet volume and atherogenic index of plasma in nondipper normotensive individuals compared to dippers. Clin Exp Hypertens. 2013;35(1):35-9. https://doi.org/1 0.3109/10641963.2012.689043.

24. Dobiásová M, Frohlich J. The plasma parameter log (TG/HDL-C) as an atherogenic index: correlation with lipoprotein particle size and esterification rate in apoB-lipoprotein-depleted plasma (FER (HDL)). Clin Biochem. 2001;34(7):583-8. https://doi.org/10.1016/50009-9120(01)00263-6.

25. Frohlich J, Dobiásová M. Fractional esterification rate of cholesterol and ratio of triglycerides to $\mathrm{HDL}$-cholesterol are powerful predictors of positive findings on coronary angiography. Clin Chem. 2003;49(11):1873-80. https:// doi.org/10.1373/clinchem.2003.022558.

26. Zhu XW, Deng FY, Lei SF. Meta-analysis of Atherogenic index of plasma and other lipid parameters in relation to risk of type 2 diabetes mellitus. Prim Care Diabetes. 2015;9(1):60-7. https://doi.org/10.1016/j.pcd.2014.03.007.

27. Zhu X, Yu L, Zhou H, Ma Q, Zhou X, Lei T, et al. Atherogenic index of plasma is a novel and better biomarker associated with obesity: a population-based cross-sectional study in China. Lipids Health Dis. 2018; 17(1):37. https://doi.org/10.1186/s12944-018-0686-8.

28. Wang $\mathrm{Q}$, Zheng $\mathrm{D}$, Liu J, Fang L, Li Q. Atherogenic index of plasma is a novel predictor of non-alcoholic fatty liver disease in obese participants: a cross-sectional study. Lipids Health Dis. 2018;17(1):284. https://doi.org/10.11 86/s12944-018-0932-0.

29. Choudhary MK, Eräranta A, Koskela J, Tikkakoski AJ, Nevalainen PI, Kähönen $M$, et al. Atherogenic index of plasma is related to arterial stiffness but not to blood pressure in normotensive and never-treated hypertensive subjects. Blood Press. 2019;28(3):157-67. https://doi.org/10.1080/08037051.2019.1583 060.

30. Wang L, Chen F, Xiaogi C, Yujun C, Zijie L. Atherogenic index of plasma is an independent risk factor for coronary artery disease and a higher SYNTAX score. Angiology. 2021;72(2):181-6. https://doi.org/10.1177/000331972094 9804.

31. Kammar-García A, López-Moreno P, Hernández-Hernández ME, Ortíz-Bueno AM, Martínez-Montaño MLC. Atherogenic index of plasma as a marker of cardiovascular risk factors in Mexicans aged 18 to 22 years. Proc (Baylor Univ Med Cent). 2020;34(1):22-7. https://doi.org/10.1080/08998280.2020.1 799479.

32. Ni W, Zhou Z, Liu T, Wang H, Deng J, Liu X, et al. Gender-and lesion number-dependent difference in "atherogenic index of plasma" in Chinese people with coronary heart disease. Sci Rep. 2017;7(1):13207. https://doi. org/10.1038/s41598-017-13267-6.

33. Lippi G, Montagnana M, Luca Salvagno G, Targher G, Cesare GG. Epidemiological association between uric acid concentration in plasma, lipoprotein(a), and the traditional lipid profile. Clin Cardiol. 2010;33(2):E7680. https://doi.org/10.1002/clc.20511.

34. Yusuf S, Reddy S, Ounpuu S, Anand S. Global burden of cardiovascular diseases: part l: general considerations, the epidemiologic transition, risk factors, and impact of urbanization. Circulation. 2001;104(22):2746-53. https://doi.org/10.1161/hc4601.099487.

35. Goliasch G, Wiesbauer F, Blessberger H, Demyanets S, Wojta J, Huber K, et al. Premature myocardial infarction is strongly associated with increased levels of remnant cholesterol. J Clin Lipidol. 2015;9(6):801-6. https://doi. org/10.1016/j.jacl.2015.08.009.

36. Khodadi E. Platelet function in cardiovascular disease: activation of molecules and activation by molecules. Cardiovasc Toxicol. 2020;20(1):1-10. https://doi.org/10.1007/s12012-019-09555-4.

\section{Publisher's Note}

Springer Nature remains neutral with regard to jurisdictional claims in published maps and institutional affiliations.
Ready to submit your research? Choose BMC and benefit from:

- fast, convenient online submission

- thorough peer review by experienced researchers in your field

- rapid publication on acceptance

- support for research data, including large and complex data types

- gold Open Access which fosters wider collaboration and increased citations

- maximum visibility for your research: over $100 \mathrm{M}$ website views per year

At BMC, research is always in progress.

Learn more biomedcentral.com/submissions 